\title{
Emergency Room Utilization for Dental Treatment: the Socioeconomic Impact of Utilizing Emergency Rooms to Treat Non-Traumatic Dental Issues
}

\author{
Darlene G 0’Brien* \\ Online Professor, USA
}

*Corresponding author: Darlene G O’Brien, Online Professor, USA.

Received Date: January 06, 2020

Published Date: January 30, 2020

\section{Introduction}

This paper reviews published articles that examine the impact of the utilization of hospital-based emergency room departments, in the United States (U.S.), to treat non-traumatic dental issues. Research has shown that oral health not only has an impact on one's systemic health but also on quality of life and economic productivity [1-3]. Most dental conditions such as dental caries, pulpal lesions or periodontal conditions can be treated routinely in a private practice or a clinic-based setting. If left untreated, these conditions can develop into more serious conditions and could even result in death [4]. Studies have shown that the usage of emergency rooms (ERs) for non-traumatic dental care has continued to increase since 2001 [5-8]. Although the Affordable Care Act (ACA) has increased access to care for some populations, disparities remain.

In addition, oral health disparities are on the rise in this country, when it comes to minorities, the poor and populations, who lack health insurance or have a greater rate of dental disease [9]. An understanding of the causes behind this increase of ER usage and its effects is crucial in the development of initiatives to improve access to care, promote preventative care and avoid the use of valuable hospital resources for nonemergency dental treatment. This paper explores the disparities in access to dental care that has led to the increase of ER usage, the impact of these disparities on our population, as well as, the economic impact on the U.S. healthcare system from the use of ERs for the treatment of non-traumatic dental issues.

\section{Literature Review}

In a qualitative study by Gift et al. [1] the results from a National Health Interview Survey were analyzed and it was found that in 1989, 164 million hours of work were missed by employed participants as a result of dental visits or issues. Females, blacks and adults aged 17-24 missed the greatest amount of time. Those who lacked dental insurance, were less educated and had a lower income were more likely to have missed work. Those in higher social classes making more money lost significantly less work hours than service workers. Nationwide, 51million hours of school were also missed, as a result of dental visits or oral problems. The most school hours were missed by females, Hispanics, participants with lower income and those without dental insurance.

In addition, a total of 41 million restricted activity days were reported for all ages nationwide with the more disadvantaged socioeconomic groups, participants lacking dental insurance and those with less than 12 years education having the most restricted activity days. Gift et al. noted that this study indicates the burden of lost work/wages, school and activity is higher in populations with low economic status and lack of insurance. Gift et al. [1], MacDougall [2] noted that a lack of dental care for people living in poverty may lead to a lower rate of employability related to missing teeth or dental deformities resulting in a further need for public assistance. Several of the studies reviewed showed a correlation between Medicaid recipients or those who lacked dental insurance and an increase in ER usage to treat non-traumatic dental problems. In a national study conducted from 2008-2010, data was collected from the Nationwide Emergency Department Sample. It was found that almost $71 \%$ of all ER visits, involving dental related issues, were from people living in low income areas. In addition, $40.5 \%$ of these visits were made involved patients without any dental insurance and an additional 30\% were covered by Medicaid [9]. 
In a three-year pediatric study by Hom et al. [6], data was collected from 110 hospitals in North Carolina. Medicaid was the source of payment $62 \%$ of the time, making it the most common form of reimbursement, in this study, each year [6]. In a 2010 study, Davis et al. [10] looked at data from seven hospitals in the Minneapolis-St. Paul metropolitan area in Minnesota. They also found that the majority of the participants in the study, who utilized the ER for non-traumatic dental issues were on public assistance, such as Medicaid or SCHIP. However, a substantial amount of visits was also coded as self-pay indicating that patients lacked dental insurance. The majority of patients utilizing the ER to treat nontraumatic dental issues were between the ages of 20 and 50. It was noted that these results reflected similar results from other studies [10]. An increase in utilization of the ER to treat nontraumatic dental problems was also seen in a 2010 New Hampshire study. Data was analyzed from the New Hampshire Emergency Department Hospital Discharge and it was found that 15-44-yearold persons were the most likely to seek dental treatment in the ER and self- pay was indicated as the major primary payer [5].

Concern about the lack of definitive care received by patients seeking non-traumatic dental care in the ER was mentioned in multiple studies that were reviewed. In the study by Anderson et al. [5], it was noted that the hospitals were not sufficiently equipped to treat non traumatic dental issues, nor did they have the staff to provide definitive dental care. The study indicated that this was an inappropriate use of hospital resources and patients who were treated in an ER setting would not necessarily receive follow up care. Without follow up treatment, the condition would likely become more serious since dental conditions are not typically selflimiting. Anderson et al. [5], Davis et al. [10] found that about 20\% of the participants had between 2-11 dental related visits to the ER. Although they couldn't determine if the patients were returning for the same dental problem, they felt this was a result of the inability of the ER to provide definitive care. Patients needed to return to the ER due to the lack of follow treatment after the initial visit or a progression of untreated underlying dental disease [10].

In a 2015 study by Okunseri et al. [7], data was analyzed from the National Hospital Ambulatory Medical Care Survey for 20072010. This is a survey of emergency and outpatient departments in hospitals throughout the 50 states. The focus of this study was to determine the rate of prescription of opiods for patients who present in the ER for non-traumatic dental issues. This study found that 50.3 percent of the patients, who presented with non-traumatic dental issues, were given an opiod prescription. The authors noted their concern that this was because ERs cannot provide routine dental care and provide definitive dental care for someone who presents with a toothache and is in need of an extraction or endodontic treatment to relieve the pain. Rather, the patient is treated with palliative care and typically receives an antibiotic and painkillers. Okusari et al. [7], Sun et al. [8] conducted a mixed-methods study, which included both quantitative and qualitative components that reflected similar sentiments.
In the quantitative portion of this study, ER claims data were obtained from 45 Oregon hospitals as well as the Oregon All Payer All Claims file. All ER visits in 2010 were included. A total of 15,081 patients were seen for non-traumatic dental issues and dental problems were the 12th most common ER diagnosis category. The study found that dental procedures were rarely performed and opoid analgesics were dispensed to $56 \%$ of the patients. In addition, it was found that more than $25 \%$ of these patients had more than 1 ER dental visit and $21 \%$ had 2-4 ER dental visits. In the qualitative portion of this study, 17 ER dental visitors were interviewed. Participants indicated that they were unable to pay for treatment at a dental office and so they sought treatment at the ER instead. Frustration was expressed over the lack of definitive care provided at the ER and that they were then referred back to a dentist for treatment they couldn't afford, leading to a vicious cycle. ER department providers and community dentists were also interviewed. They were in agreement that the care provided in ERs is palliative. Concern was expressed over the return visits to the ER and repeat prescriptions for pain medication [8].

The cost effectiveness of using the ER to treat non-traumatic dental issues was raised in some of the studies reviewed. Singhal et al. [3] conducted an interrupted time series study with controlled variables. The target population was people 21 or older, who were enrolled in Medicaid, during 2006-2011. The objective of the study was to examine the impact of the elimination of dental coverage for adults on Medicaid, in California. During this time period, 113,309 Medicaid adult enrollees visited the ER for dental problems. After the elimination of Medicaid adult dental benefits, there was $32.3 \%$ increase in ER dental visits by this group. As a result, this increased the average that California Medicaid program spent by an additional 1.25 million for ER visits. Although this represents a 66-million-dollar savings for the state, the authors noted that the additional ER costs did not represent a resolution to the dental problem which would likely incur more costs. In addition, costs of hospital admissions, physician visits, loss of productivity and quality of life should be considered [3]. A PEW Trust study found that the average cost of a Medicaid enrollee's inpatient hospital care for the treatment of dental issue far exceeds what it would cost for preventative treatment received in a dental office by nearly ten times. MacDougall [2], Davis et al [10] found that the cumulative charges by 5 Minnesota hospitals in a one-year period equaled 4,743,519 dollars. The authors noted that many of these charges were associated with visits that were avoidable or a result of incomplete treatment and a lack of follow up care resulting in repeat ER visits [10].

\section{Strengths and Limitations of the Literature}

The majority of the research reviewed involved a retrospective analysis of data collected from multiple databases which means that causality could not be proven. Sun et al. [8] provided us some insight into causality but the cohort, for the qualitative portion of their study was small. The data collected from ERs was helpful in establishing the reasons for ER visits, but it usually involved 
dental diagnoses codes that were entered by ER physicians. Since "unspecified teeth and supporting structures" tended to be the most common diagnosis in all of the studies, which analyzed these codes, this leaves open the possibility of misclassification bias. Several of the studies, that were reviewed, looked at ER visits on a statewide level and therefore cannot be used to predict nationwide trends. However, when comparing these studies, they all tended to have similar results and conclusions, indicating national trends and strengthening the validity of their findings.

The full scope of the economic impact on this country is unclear. The costs of treatment from all nontraditional venues that are used to treat non-traumatic dental issues must be taken into consideration along with the impact on productivity and the quality of life. In Addition, further research needs to be done on this topic to determine specific barriers that result in the use of the ER for dental care as well as to establish the success or lack of success of interventions.

\section{US Healthcare System Implications}

The US government needs to consider the implications of the access to care issue in this country and take further action to reduce barriers to oral healthcare. Because oral health is an integral part of systemic health, barriers to care have the potential to affect the quality of life and economic productivity of US citizens. Additionally, usage of the ER for non-emergency dental treatment is a costing millions per year and is not an efficient utilization of a hospital's resources. Preventative programs should be at the heart of any initiative taken. Dental disease is largely preventable and should not escalate into an urgent care issue. School based programs are key and certainly, at risk populations should be targeted. Increasing dental knowledge regarding the importance of oral health, preventative care and how to access care is particularly important in at risk populations. I believe it would be helpful for hospitals to pair with dental schools, as an externship site, to include dental professionals on hospital staff. Unfortunately, this could be difficult for some hospitals due to their geographic location. All hospitals should develop a relationship with local dental clinics or other dental resources so that patients can be referred for more definitive treatment. It would also be helpful for emergency room staff to be familiar with their state's Medicaid coverage in order to facilitate the referral of patients to receive definitive dental treatment. Medicaid benefits for adults should be expanded nationwide but provider availability must be addressed. Reimbursement for Medicaid providers should be increased to address the access to care issue due to limited providers. Finally, if legislation that supports the role of midlevel dental providers is implemented in every state, it would increase access to care by increasing dental providers that except Medicaid and providing more financially feasible treatment for the uninsured.

\section{Conclusion}

The trend of ER usage for dental problems is increasing nationwide. A significant number of the people, who are Medicaid recipients or uninsured, utilize the ER to treat non-traumatic dental issues that could and should be treated in an office setting. In particular, young adults, seemed to be the most apt to use this venue to address their dental needs. The research reviewed points to a lack of insurance and dental knowledge, as well as barriers to access to care as possible factors responsible for the misuse of emergency departments for dental care. Emergency rooms are not equipped to address non traumatic dental issues and provide definitive care. Instead, patients often receive palliative treatment involving the prescription of antibiotics and opiod analgesics. Given the concern, regarding the over prescribing of antibiotics and the current national opiod addiction crisis, this is far from optimal. In addition, the cost of a hospital-based visit greatly outweighs the cost of preventative care in an office or clinic setting.

More research needs to be done, regarding this matter, to determine exact causative factors. The government must consider the cost effectiveness and socioeconomic impact of reducing dental care benefits. Furthermore, they must make it a priority to formulate initiatives that would increase access to routine preventative dental care and definitive treatment, when needed, to reduce the utilization of emergency departments to treat dental problems. Medicaid benefits for adults should be expanded nationwide, provider reimbursement for Medicaid should be increased and the role of midlevel dental providers should be implemented in every state.

\section{Acknowledgement}

None.

\section{Conflict of Interest}

No conflict of interest.

\section{References}

1. Gift HC, Reisine ST, Larach DC (1992) The social impact of dental problems and visits. Am J Public Health 82(12): 1663-1668.

2. MacDougall H (2016) Dental disparities among low-income American adults: a social work perspective. Health Soc Work 41(3): 208-210.

3. Singhal A, Caplan DJ, Jones MP, Momany ET, Kuthy RA, et al. (2015) Eliminating Medicaid adult dental coverage in California led to increased dental emergency visits and associated costs. Health Aff 34(5): 749-756.

4. Allareddy V, Rampa S, Min Kyeong L, Allareddy V, Nalliah RP (2014) Hospital-based emergency department visits involving dental conditions: Profile and predictors of poor outcomes and resource utilization. J Am Dent Assoc 145(4): 331-337.

5. Anderson L, Cherala S, Traore E, Martin NR (2011) Utilization of hospital emergency departments for non-traumatic dental care in New Hampshire 2001-2008. J Community Health 36(4): 513-516.

6. Hom JM, Burgette LF, Lee JY (2013) The effect of North Carolina hospital payor mix on dental-related pediatric emergency room utilization. J Public Health Dent 73(4): 289-296.

7. Okunseria C, Dionne RA, Gordon SM, Okunseria E, Szabod A (2015) Prescription of opioid analgesics for nontraumatic dental conditions in emergency departments. Drug Alcohol Depend 156: 261-266.

8. Sun BC, Chi DL, Schwarz E, Milgrom P, Yagapen A, et al. (2015) Emergency department visits for nontraumatic dental problems: a mixed-methods study. Am J Public Health 105(5): 947-955. 
9. Allareddy V, Nalliah RP, Haque M, Johnson H, Tech SB, et al. (2014) Hospital-based emergency department visits with dental conditions among children in the United States: nationwide epidemiological data. Pediatr Dent 36(5): 393-399.
10. Davis EE, Deinard AS, Maïga EH (2010) Doctor, my tooth hurts: the costs of incomplete dental care in the emergency room. J Public Health Dent $70(3): 205-210$. 